\title{
PA-091 MEETING FIELD-BASED CHALLENGES: INNOVATIVE APPROACHES TO COLLECTING DRY BLOOD SPOT SAMPLES IN THE COMMUNITY
}

\author{
Kombatende Sikombe, ${ }^{1}$ Cardinal Hantuba, ${ }^{1}$ Nancy Czaicki, ${ }^{2}$ Elvin Geng, ${ }^{3}$ \\ Charles Holmes, ${ }^{1}$ Kalo Musukuma, ${ }^{1}$ Sandra Simbeza, ${ }^{1}$ Paul Somwe, ${ }^{1}$ \\ Izukanji Sikazwe'. ' ${ }^{1}$ CIDRZ, Zambia; ${ }^{2}$ University of California, Berkley, United States \\ of America; ${ }^{3}$ University of California, San Francisco, United States of America
}

\subsection{6/bmjgh-2016-000260.123}

Background Community collection of dry blood spots (DBS) is ideal to capture viral load results from HIV-positive, patients lost to follow-up (LFTU) in order to monitor their health. We sought to optimise protocols for high-quality community-based DBS collection in resource-constrained settings such as Zambia. Methods As part of a nested case-control study, we trained 23 non-medical interviewers who collected DBS from LTFU patients in rural and urban Lusaka. We visited another Zambian community-based DBS study improving upon their approach through team-based problem solving methods. We evaluated our innovation through field observations, bi-weekly meetings, interviewer reports, and two debriefing meetings. The laboratory assessed DBS quality for testing validity.

Results We transformed a first-aid box into a phlebotomy box to keep DBS contamination free and in ambient temperature. A styrofoam partition separated the DBS drying rack glued to one side of the box from phlebotomy supplies and kept DBS cards horizontal during transportation. Interviewers collected 229 DBS (60.6\%) in participant homes or place of their choice with 149 refusals. DBS was air-dried in an area free of direct sunlight, water, insects and dust for a few minutes so blood was not flowing when placed on the rack. DBS was taken to the nearest health facility for further drying using public transport, or study motorbikes fitted with a custom made carrier to hold the box horizontal. The laboratory did not report any blotted or double spotted DBS cards. Barriers included privacy, visibility and awkward box size.

Conclusions We optimised community-based DBS collection in Zambia using non-medical staff and an innovative, low-cost light-weight phlebotomy box to transport DBS without contamination at ambient temperature. While we successfully collected DBS from $60.6 \%$ of found LTFU patients, concerted efforts are needed to re-engage LTFU patients who refuse HIV-related procedures even when made conveniently available. 